\title{
Exact metric around a wiggly cosmic string
}

\author{
N. Özdemir \\ ITU, Faculty of Sciences and Letters, Department of Physics, 80626 \\ Maslak, and Feza Gürsey Institute, 81220 Çengelköy; Istanbul, Turkey
}

\begin{abstract}
The exact metric around a wiggly cosmic string is found by modifying the energy momentum-tensor of a straight infinitely thin cosmic string to include an electric current along the symmetry axis.
\end{abstract}


Cosmic strings have widely been studied in the literature over the last years [1]. There are two main reasons for active investigations in the theory of cosmic strings; the first is stimulated by cosmological implications of the cosmic strings, as the string network in the early Universe may provide viable density fluctuations for the formation of the large-scale structure [2]. The second reason is due to unusual gravitational effects of cosmic strings [1][5], since the space-time geometry around a straight cosmic string is locally flat, but globally it is identical to the geometry of a cone [6]. Numerical simulations of the string network have shown the presence of small-scale perturbations (wiggles) running along a long straight cosmic strings [7]. These wiggles should affect on the energy density and tension of the strings and can be taken into account in the study of cosmological and physical consequences of the cosmic string network. One way how to take into consideration the effect of wiggles is to average over small-scale perturbations on a long string. It leads to an effective energy density $\mu$ and an effective tension $T$, which satisfy to the equation of state

$$
\mu T=\mu_{0}^{2}
$$

where $\mu_{0}$ is the energy density per unit length of an unperturbed cosmic string. With the equation of state (1) the energy-momentum tensor of a wiggly cosmic string oriented along the $z$-axis can be approximated by the equation

$$
T_{\mu}^{\nu}=\operatorname{diag}(\mu, 0,0, T) \delta(x) \delta(y) .
$$

Using this energy-momentum tensor as a source in the right-hand-side of the linearized Einstein field equations one can find the gravitational field around a wiggly cosmic string [8]. This result is given by

$$
\begin{aligned}
& h_{00}=h_{33}=4 G(\mu-T) \ln r \\
& h_{11}=h_{22}=4 G(\mu+T) \ln r
\end{aligned}
$$

where we can easily see that the wiggly cosmic string produces a newtonian force. However it becomes not possible to extend the solution (3) to full relativistic case, that is to find the exact solution of the Einstein field equations with the energy-momentum tensor (2).

In this note we shall find the exact metric around a wiggly cosmic string by modifying the energy momentum-tensor (2). We shall suppose that the 
wiggly string is still infinitely thin to be approximated by the $\delta$-function like source, however unlike (2), its energy-momentum tensor contains additional components in the $x$ and $y$ directions. Thus we shall consider the energymomentum tensor

$$
T_{\mu}^{\nu}=\operatorname{diag}(\mu+a, b, b, T+a) \delta(x) \delta(y)
$$

where $a$ and $b$ are arbitrary constant parameters, which can be related to the density of an electric current carried by the string along the $z$-axis. We start with the metric in cylindrical coordinates

$$
d s^{2}=e^{2 A} d t^{2}-e^{2 B} d z^{2}-e^{2 C} d r^{2}-r^{2} e^{2 L} d \varphi^{2}
$$

where $A, B, C$ and $L$ are the functions of distance $r$. Then the Einstein field equations with the source (4) are reduced to the following set of equations

$$
\begin{array}{r}
\left(r B_{, r} e^{A+B-C+L}\right)_{, r}=-4 \pi(\mu-T+2 b) \frac{\delta(r)}{r} \\
\left(\left(r L_{, r}+1\right) e^{A+B-C+L}\right)_{, r}=-4 \pi(\mu+T+2 a) \frac{\delta(r)}{r} \\
e^{A+B-C+L}\left(r L_{, r r}+2 L_{, r}+r L_{, r}^{2}-C_{, r}\left(1+r A_{, r}+r B_{, r}+r L_{, r}\right)\right. \\
\left.+r A_{, r}^{2}+r B_{, r}^{2}+r A_{, r r}+r B_{, r r}\right)=-4 \pi(\mu+T+2 a) \frac{\delta(r)}{r}
\end{array}
$$

where the subscript,$r$ denotes derivative with respect to $r$. The general solution which are subject to these equations has the form

$$
\begin{aligned}
A & =2(\mu-T-2 b) \ln r \\
B & =-2(\mu+T+2 b) \ln r \\
L & =-[2(\mu+T+2 a)+1] \ln r \\
C & =-[2(\mu+T+4 b+2 a)+1] \ln r
\end{aligned}
$$

where additive constants of integration are taken to be zero. Finally, the metric is given by the expression

$$
d s^{2}=\left(r^{4(\mu-T-2 b)} d t^{2}+r^{-4(\mu-T+2 b)} d z^{2}\right)-r^{-4(\mu+T+4 b+2 a)-2}\left(d r^{2}+r^{2+16 b} d \varphi^{2}\right)
$$


which describes the gravitational field near a wiggly cosmic string, carrying a current on the $z$-axis. Indeed, the conservation of the energy momentum tensor gives the relation

$$
8 b^{2}+b(1-8 a-4 b(\mu+T))+2(\mu-T)^{2}=0
$$

which enables us to reduce two unkwown constants $a$ and $b$ in the metric to one of them, which in turn, as it was mentioned above, can be related to the density of an electric current along the symmetry axis [9]. We note that when the electric current vanishes $(a \rightarrow 0, b \rightarrow 0)$ the metric (13) reduces to its linearized limit [8].

We have found the exact solution of the Einstein field equations which determines the gravitational field of a long wiggly cosmic string carrying an electric current on it. This is a generalization of a corresponding linearized solution to the full relativistic case. It requires the presence of a current on the symmetry axis of the wiggly cosmic string.

\section{Acknowledgement}

I thank A. N. Aliev for valuable discussions.

\section{References}

[1] Vilenkin A and Shellard E P S Cosmic Strings and Other Topological Defects (1994) (Cambridge Univ. Press Cambridge, England)

[2] Zel'dovich Ya. B. (1980). Monthly Notes R. Astron.Soc. D192, 663.

[3] Aliev A.N. and Galt'sov D.V. (1989). Ann. Phys. 193, 142.

[4] Aliev A.N. and Galt'sov D.V. (1988). Sov. Astron.Lett. 1448.

[5] Aliev A N, Hortacsu M and Özdemir N (1997) Class. Quant. Grav. 14 3215

[6] Hiscock W A (1985) Phys. Rev. D31 3288.

[7] Vachaspati T, Vilenkin A (1991)Phys. Rev. Lett. 671057.

[8] Vilenkin A (1990) Phys. Rev. D41 3038. 
[9] Moss I and Poletti S. (1987) Phys. Lett. B 19934. 